\section{Performance of timing resistive plate chambers with relativistic neutrons from 300 to $1500 \mathrm{MeV}$}

To cite this article: A. Blanco et al 2015 JINST 10 C02034

View the article online for updates and enhancements.

\section{Related content}

- $\frac{\text { Performance of timing Resistive Plate }}{\text { Chambers with protons from } 200 \text { to } 800}$
$\frac{\text { MeV }}{\text { J. Machado, J. Adamczewski-Musch, A. }}$
Blanco et al.
- GEANT4 simulation of gamma rav in a
$\frac{\text { double-gap resistive plate chamber }}{\text { J. T. Rhee, M. Jamil, Steve Hall et al. }}$
- Design and test of a Multi-gap Resistive
$\frac{\text { Plate Chamber with Long readout-strip }}{\text { (LMRPC) }}$
Sun Yong-Jie, Li Cheng, Zhou Yi et al.

\section{Recent citations}

\author{
Multi-purpose trigger system for medium \\ particle detector devices \\ F. Clemêncio et al \\ Detectors and Concepts for sub-100 ps \\ timing with gaseous detectors \\ D. González-Díaz et al
}




\section{Performance of timing resistive plate chambers with relativistic neutrons from 300 to $1500 \mathrm{MeV}$}
A. Blanco, ${ }^{a, 1}$ J. Adamczewski-Musch, ${ }^{d}$ K. Boretzky, ${ }^{d}$ P. Cabanelas, ${ }^{b, e}$ L. Cartegni, ${ }^{b}$
R. Ferreira Marques, ${ }^{a, f}$ P. Fonte, ${ }^{a, g}$ J. Fruehauf, ${ }^{d}$ D. Galaviz, ${ }^{b}$ M. Heil, ${ }^{d}$
A. Henriques, ${ }^{b}$ G. Ickert, ${ }^{d}$ D. Körper ${ }^{d}$ L. Lopes, ${ }^{a}$ M. Palka, ${ }^{h}$ A. Pereira, ${ }^{a}$ D. Rossi, ${ }^{d}$
H. Simon, ${ }^{d}$ P. Teubig, ${ }^{b}$ M. Traxler, ${ }^{d}$ P. Velho, ${ }^{b}$ S. Altstadt, ${ }^{i}$ L. Atar,${ }^{j}$ T. Aumann, ${ }^{j}$
D. Bemmerer, ${ }^{k}$ C. Caesar, ${ }^{j}$ A. Charpy, ${ }^{l}$ Z. Elekes, ${ }^{k}$ E. Fiori, ${ }^{m, n}$ I. Gasparic, ${ }^{j}$
J. Gerbig, ${ }^{i}$ K. Göbel, ${ }^{i}$ T. Heftrich, ${ }^{i}$ M. Heine, ${ }^{j}$ A. Heinz, ${ }^{l}$ M. Holl, ${ }^{j}$ A. Ignatov, ${ }^{j}$
J. Isaak, ${ }^{m, n}$ H. Johansson, ${ }^{l}$ A. Kelic-Heil, ${ }^{d}$ C. Lederer,${ }^{i}$ S. Lindberg, ${ }^{l}$ B. Löher, ${ }^{m, n}$
J. Machado, ${ }^{b, c}$ J. Marganiec, ${ }^{m, n}$ M. Martensson, ${ }^{l}$ T. Nilsson, ${ }^{l}$ V. Panin, ${ }^{j}$ S. Paschalis, ${ }^{j}$
M. Petri, ${ }^{j}$ R. Plag, ${ }^{d}$ M. Pohl, ${ }^{i}$ G. Rastrepina, ${ }^{i}$ R. Reifarth, ${ }^{i}$ T. P. Reinhardt, ${ }^{n}$ M. Röder, ${ }^{o}$
D. Savran, ${ }^{m, n}$ H. Scheit, ${ }^{j}$ P. Schrock, ${ }^{j}$ J. Silva, ${ }^{m, n}$ D. Stach, ${ }^{k}$ F. Strannerdahl, ${ }^{l}$
R. Thies, ${ }^{l}$ A. Wagner, ${ }^{k}$ F. Wamers ${ }^{d}$ and M. Weigand ${ }^{i}$ for the R3B Collaboration

${ }^{a}$ LIP, Laboratório de Instrumentação e Física Experimental de Partículas, 3004-516 Coimbra, Portugal
${ }^{b}$ Centro de Física Nuclear da Universidade de Lisboa, CFNUL, 1649-003 Lisboa, Portugal
${ }^{c}$ Centro de Física Atómica, CFA, Departamento de Física, Faculdade de Ciências e Tecnologia, FCT,
Universidade Nova de Lisboa, 2829-516 Caparica, Portugal
${ }^{d}$ GSI, Helmholtzzentrum für Schwerionenforschung, Darmstadt, D-64291 Darmstadt, Germany
${ }^{e}$ Departamento de Física de Partículas, Universidade de Santiago de Compostela,
15706 Santiago de Compostela, Spain
${ }^{f}$ Departamento de Física, Universidade de Coimbra, 3004-516 Coimbra, Portugal
${ }^{g}$ ISEC-Instituto Superior de Engenharia de Coimbra, 3031-199 Coimbra, Portugal
${ }^{h}$ Uniwersytet Jagielloński, Cracow, Poland
${ }^{i}$ Goethe Universitä Frankfurt am Main, 60438 Frankfurt am Main, Germany
${ }^{j}$ Institut für Kernphysik Technische UniversitätDarmstadt, 64289 Darmstadt, Germany
${ }^{k}$ Helmholtz-Zentrum Dresden-Rossendorf, D-01328 Dresden, Germany
${ }^{l}$ Fundamental Fysik, Chalmers Tekniska Högskola, S-412 96 Göteborg, Sweden

\footnotetext{
${ }^{1}$ Corresponding author.
} 
${ }^{m}$ ExtreMe Matter Institute EMMI and Research Division, GSI Helmholtzzentrum für chwerionenforschung GmbH, 64291 Darmstadt, Germany

${ }^{n}$ Frankfurt Institute for Advanced Studies FIAS, Frankfurt am Main, Germany

${ }^{o}$ Institut für Kern- und Teilchenphysik, Technische Universität Dresden, 01069 Dresden, Germany

E-mail: albertor@coimbra.lip.pt

ABSTRACT: A prototype composed of four resistive plate chamber layers has been exposed to quasi-monoenergetic neutrons produced from a deuteron beam of varying energy (300 to $1500 \mathrm{AMeV}$ ) in experiment $S 406$ at GSI, Darmstad, Germany. Each layer, with an active area of about $2000 \times 500 \mathrm{~mm}^{2}$, is made of modules containing the active gaps, all in multigap construction. Each gap is defined by $0.3 \mathrm{~mm}$ nylon mono-filaments positioned between $2.85 \mathrm{~mm}$ thick float glass electrodes. The modules are operated in avalanche mode with a non-flammable gas mixture composed of $90 \% \mathrm{C}_{2} \mathrm{H}_{2} \mathrm{~F}_{4}$ and $10 \% \mathrm{SF}_{6}$. The signals are readout by a pick-up electrode formed by 15 copper strips (per layer), spaced at a pitch of $30 \mathrm{~mm}$, connected at both sides to timing front end electronics. Measurements of the time of flight jitter of neutrons, in the mentioned energy range, point to a contribution of the resistive plate chamber in the order of $150 \mathrm{ps}$, independent of the neutron energy.

KEYWORDS: Resistive-plate chambers; Instrumentation and methods for time-of-flight (TOF) spectroscopy; Neutron detectors (cold, thermal, fast neutrons); Particle detectors 


\section{Contents}

1 Introduction 1

2 Experimental setup $\quad 1$

2.1 RPC prototype 1

$\begin{array}{lll}2.2 & \text { Experiment S406 }\end{array}$

3 Methods 4

3.1 Timing resolution $\quad 4$

3.2 Neutron selection $\quad 5$

4 Results $\quad 7$

$\begin{array}{lll}4.1 & \text { RPC timing resolution } & 7\end{array}$

5 Conclusions $\quad 8$

\section{Introduction}

With the new Facility for Antiproton and Ion Research (FAIR) in Darmstadt, Germany, higher beam intensities, brilliant beam quality and higher beam energies of secondary radioactive beams will be achievable. In this context, the existing experimental setup has to be upgraded and in particular, the neutron Time of Flight (TOF) detector of the $\mathrm{R}^{3} \mathrm{~B}$ collaboration [1], NeuLAND [2]. The design goals of NeuLAND have been chosen to match the momentum resolution of the charged fragments of $\Delta p / p \approx 10^{-3}$. To achieve this momentum resolution a timing resolution $\sigma_{t}<100 \mathrm{ps}$ and a position resolution $\sigma_{x, y, z} \approx 1 \mathrm{~cm}$ for flight paths in the range from 10 to $35 \mathrm{~m}$ is required. In Autumn 2012, quasi-monoenergetic neutrons were produced from a deuteron beam in experiment $S 406$ to calibrate the neutron detector LAND [3] and to study the performance of the NeuLAND prototype. During this experiment, a prototype based on timing Resistive Plate Chambers (tRPCs), considered during the R\&D phase of the NeuLAND detector and previously proposed [4], suggesting very satisfactory efficiency and momentum resolution, was also exposed to neutron beams of various energies, from 300 to $1500 \mathrm{MeV}$. The particular aspect of this concept is that the glass electrodes which define the gas gaps within the RPC act also simultaneously as converter material to detect the relativistic neutrons.

\section{Experimental setup}

\subsection{RPC prototype}

The RPC prototype was built based on sensitive modules, see figure 1. A sensitive module consists of a plastic (poly-methyl methacrylate, PMMA) tight box, which encloses and confines the gas gaps 

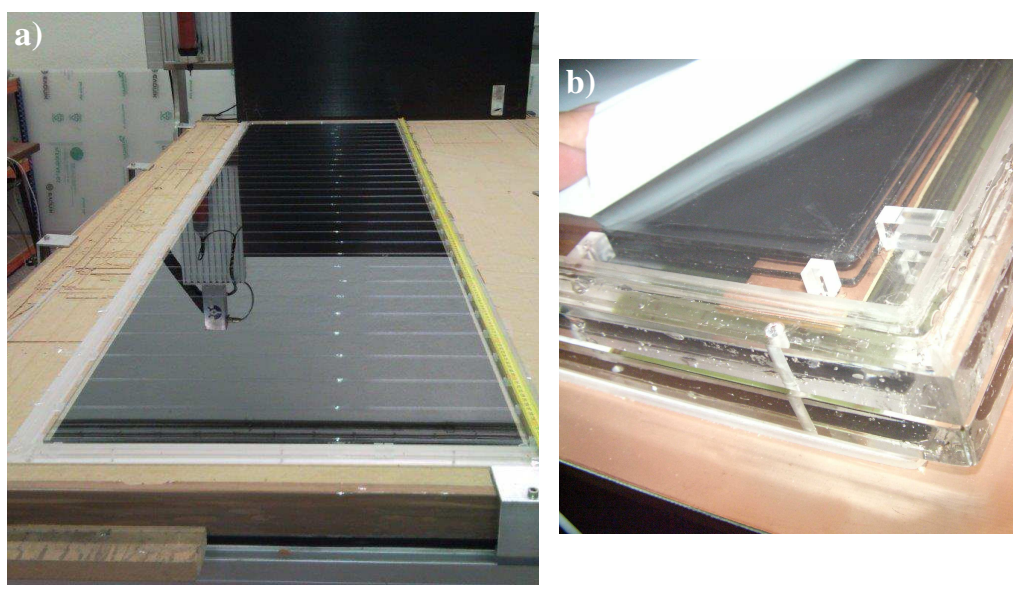

Figure 1. a) A sensitive module consists of a plastic (PMMA) tight box enclosing the HV and glass electrodes. b) Detail view of one of the gas tight box corner showing the HV electrode (black surface), glass electrodes and spacers to hold the stack inside the box.

and the glass and High Voltage (HV) electrodes. Inside this box, the gas gaps, are defined between $2.85 \mathrm{~mm}$ thick float glass ${ }^{1}$ electrodes of about $2000 \times 500 \mathrm{~mm}^{2}$ separated by $0.3 \mathrm{~mm}$ nylon monofilaments. The HV electrodes, made up of a resistive surface layer ${ }^{2}$ are applied to the outer glass surface with airbrush techniques. The plastic box is only equipped with feed-throughs for gas and HV.

A RPC layer is composed of two identical sensitive modules, read out by a pick-up electrode, made from FR4 Printed Circuit Board (PCB) with a thickness of $1.5 \mathrm{~mm}$, equipped with 15 copper strips, $29 \mathrm{~mm}$ wide and spaced at a pitch of $30 \mathrm{~mm}$, located between the modules. Each layer is enclosed in an aluminum case to guarantee the electromagnetic isolation from the environment. The prototype consists of four of such RPC layers, $L_{1}, L_{2}, L_{3}$ and $L_{4}$ (see schematic illustration in figure 2.a.) Half of the layers, $L_{1}$ and $L_{2}$, are built from modules with two gaps (4 gaps in total), while $L_{3}$ and $L_{4}$, are built from modules with five gaps (10 gaps in total), all in multigap construction [5]. They are operated in the vertical position (see figure 2.b) with a non-flammable gas mixture composed of $90 \% \mathrm{C}_{2} \mathrm{H}_{2} \mathrm{~F}_{4}$ and $10 \% \mathrm{SF}_{6}$, under a continuous gas flow of $15 \mathrm{~cm}^{3} / \mathrm{min} /$ module and at a pressure slightly under atmospheric pressure to define correctly the gap width.

The 60 strips of the prototype are read out at both ends by timing Front End Electronics (FEE) with a timing accuracy of approximately $40 \mathrm{ps}$ sigma [6]. The later, together with the corresponding Low Voltage Power Supply (LVPS) and service boards [7, 8] were borrowed from the RPC-TOF Wall [9] group of the HADES collaboration [10].

The Data Acquisition System consists basically of a TRB (Trigger Readout Board) [11], while data gathering is performed by standard HADES event builder software. Additionally, the RPC DAQ has been synchronized with the MBS-based (Multi Branch System) [12] NeuLAND DAQ by means of a synchronization message from a VOLUM4 [13] module to the TRB. This allows the offline synchronization of both data streams for later data analysis.

\footnotetext{
${ }^{1}$ Bulk resistivity of approximately $10^{12} \Omega \mathrm{cm}$ at $25^{\circ} \mathrm{C}$.

${ }^{2}$ Based on an artistic acrylic paint.
} 

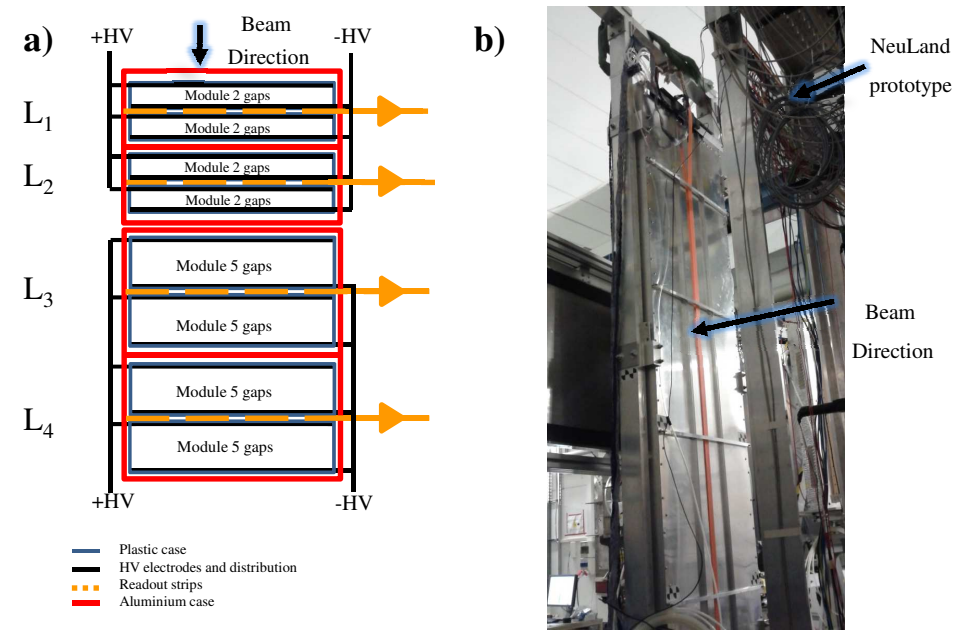

Figure 2. a) Schematic of the prototype consisting in four RPC layers. $L_{1}$ and $L_{2}$ (4-gaps RPC), are built from two sensitive modules with two gaps, while $L_{3}$ and $L_{4}$ (10-gaps RPC) are built from two modules with five gaps, all in multigap construction. b) RPC prototype standing up in the vertical position in the experimental hall.

Per layer, the prototype measures the following quantities: the time (calculated as average of times at both ends of the strip with highest charge corrected by charge and position walks), position along the strips (calculated as difference of times at both ends of the fired strips), position across the strips (calculated as mean of the position of the fired strips weighted by charge) and depth of interaction (calculated as the fired layer). In order to provide an accurate timing from the prototype, a precise calibration of the individual time of each strip was performed.

Figure 3 shows the response of the prototype to $800 \mathrm{MeV}$ protons (1.75 times more ionizing than Minimum Ionizing Particles (MIPS)) as a function of the HV/gap. This figure is a compilation
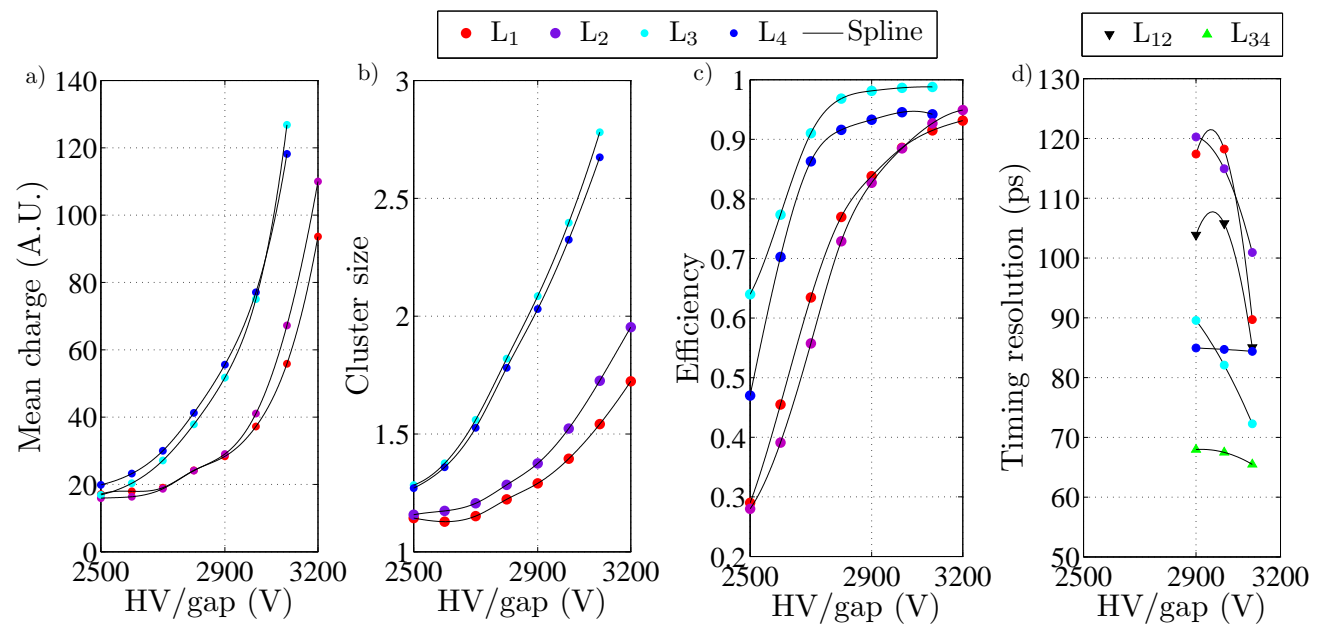

Figure 3. Prototype characterization a) mean charge, b) cluster size, c) efficiency and d) timing resolution as a function of the HV/gap for $800 \mathrm{MeV}$ protons. Compiled from [14]. 


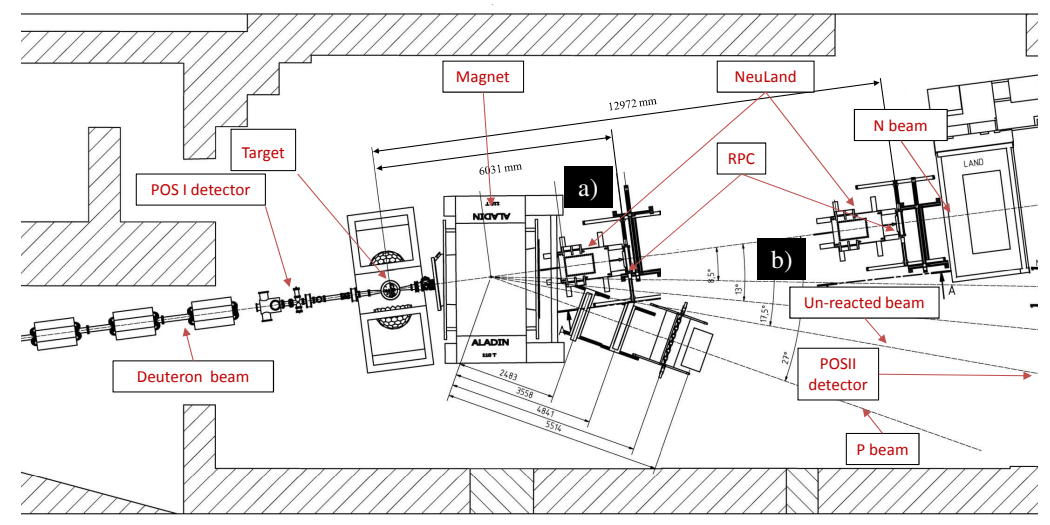

Figure 4. S406 schematic setup. From left to right, the POSI (start) detector, the target, the magnet, the Neuland prototype and, just after it, the RPC prototype standing up at a) $6.03 \mathrm{~m}$ and b) $12.97 \mathrm{~m}$ from the target.

of results from reference [14] where a comprehensive explanation can be found. The HV/gap working point for the data taking was $2900 \mathrm{~V} / \mathrm{gap}$. This value is clearly inadequate for $L_{1}$ and $L_{2}$ (lower efficiency and worse timing resolution when compared with $L_{3}$ and $L_{4}$ ) but unfortunately this characterization was only available after the experiment. Typical dark current and counting rate (at $2900 \mathrm{~V} / \mathrm{gap}$ and $25^{\circ} \mathrm{C}$ ) are $5 \mathrm{pA} / \mathrm{cm}^{2}$ gap and $0.06 \mathrm{~Hz} / \mathrm{cm}^{2}$ gap, both the 2-gaps and 5-gaps modules, which gives $20 \mathrm{pA} / \mathrm{cm}^{2}$ and $0.24 \mathrm{~Hz} / \mathrm{cm}^{2}$ for the 4-gaps layers and $50 \mathrm{pA} / \mathrm{cm}^{2}$ and $0.6 \mathrm{~Hz} / \mathrm{cm}^{2}$ for the 10-gaps layers.

\subsection{Experiment $\mathrm{S406}$}

A deuteron beam, in the energy range from 300 to $1500 \mathrm{AMeV}$, has been used to produce quasimono-energetic neutron and proton beams, derived from breakup or quasifree scattering reactions of the deuterons on a $\mathrm{CH}_{2}$ and $\mathrm{C}$ solid targets. Figure 4 shows a schematic of the $S 406$ setup. From left to right, along the deuteron beam line, one has the POSI detector (a fast scintillator $20 \times 20 \times 100 \mathrm{~mm}^{3}$, readout at both sides by two fast photomultipliers, which provides the start time) and the target surrounded by the crystal ball (a scintillator based detector covering almost $4 \pi$ solid angle). They are followed by the large gap dipole magnet ALADIN (A Large Acceptance DIpole magNet), used to bend the charged particles to their respective branches. At this point, neutrons continue in the forward direction, while protons and un-reacted deuteron beam are bent. Following in the forward direction along the neutron line, there is the Neuland prototype [2] and just after it the RPC prototype. Both detector prototypes were tested at two different distances from the target, $L, 6.03 \mathrm{~m}$ and $12.97 \mathrm{~m}$ for the RPC (see figure 4). Also following downstream, in the un-reacted beam branch, there is another fast scintillator, POSII, with the same characteristics as POSI, which provides a stop time.

\section{Methods}

\subsection{Timing resolution}

The time of flight between the POSI detector and the $i^{\text {th }}$ RPC layer, $x T O F_{i}$, is calculated as

$$
x T O F_{i}=T_{\mathrm{POS}_{\mathrm{I}}}-T_{R P C_{i}},
$$


where $x$ can be $n$, refereed to neutrons, or $\bar{n}$, refereed to other particles (see section 3.2), $T_{\mathrm{POS}_{\mathrm{I}}}$ and $T_{R P C_{i}}$ are the time from POSI detector and the $i^{\text {th }}$ RPC layer respectively. In order to characterize the resulting non gaussian distribution, the standard deviation, $\sigma\left(x T O F_{i}\right)$, of a gaussian fit within $\pm 1.5 \sigma$ around the mean of the original distribution is calculated. The three main contributions to $\sigma\left(n T O F_{i}\right)$ are the time jitter introduced by the POSI detector, $\sigma\left(T_{\mathrm{POS}_{\mathrm{I}}}\right)$, the time jitter introduced by the $i^{\text {th }}$ RPC layer, $\sigma\left(T_{R P C_{i}}\right)$ and the neutron time of flight jitter due to the internal motion of the neutron inside the deuteron $\sigma\left(T_{n}\left(E_{n}, L\right)\right)$, which depends on both the neutron energy, $E_{n}$, and the distance to the target, $L$.

- $\sigma\left(T_{\mathrm{POS}_{\mathrm{I}}}\right)$ is evaluated by calculating the time difference between POSI and POSII scintillators, $\Delta\left(T_{\mathrm{POS}}\right)=T_{\mathrm{POS}_{\mathrm{I}}}-T_{\mathrm{POS}_{\mathrm{II}}}$, for the unreacted deuteron beam. Assuming equal contributions from both detectors, $\sigma\left(T_{\mathrm{POS}_{\mathrm{I}}}\right)=\sigma\left(\Delta\left(T_{\mathrm{POS}}\right)\right) / \sqrt{2}$, where $\sigma\left(\Delta\left(T_{\mathrm{POS}}\right)\right)$ is the standard deviation of a gaussian fit within $\pm 1.5 \sigma$ around the mean of the original $\Delta\left(T_{\mathrm{POS}}\right)$ distribution.

- $\sigma\left(T_{R P C_{i}}\right)$ is evaluated by calculating the time difference between two given layers, $i$ and $j$, of the RPC, $\Delta\left(T_{R P C_{i j}}\right)=T_{R P C_{i}}-T_{R P C_{j}}$. Assuming the same contribution from both layers, $\sigma\left(T_{R P C_{i}}\right)=\sigma\left(\Delta\left(T_{R P C_{i j}}\right)\right) / \sqrt{2}$, where $\sigma\left(\Delta\left(T_{R P C_{i j}}\right)\right)$ is the standard deviation of a gaussian fit within $\pm 1.5 \sigma$ around the mean of the original $\Delta\left(T_{R P C_{i j}}\right)$ distribution.

- $\sigma\left(T_{n}\left(E_{n}, L\right)\right)$ is calculated by considering a gaussian distribution in momentum space with $\sigma\left(p_{n}\right)=\sqrt{m_{n} S_{n}}=45.72 \mathrm{MeV} / \mathrm{c}$, where $p_{n}$ is the neutron momentum, $m_{n}$ is the neutron rest mass and $S_{n}$ is the binding energy of the neutron inside the deuteron. The resulting momentum jitter is not constant and also becomes relatively important with decreasing beam energy. The results for $\sigma\left(T_{n}\left(E_{n}, L\right)\right)$, calculated for the two different distances to the target and as a function of the incident deuteron beam energy, are presented in table 1.

Thus, the $\sigma\left(n T O F_{i}\right)$ can be calculated by quadratic addition of the different contributions

$$
\sigma\left(n T O F_{i}\right)_{\mathrm{cal}}=\sqrt{\sigma\left(T_{\mathrm{POS}_{\mathrm{I}}}\right)^{2}+\sigma\left(T_{R P C_{i}}\right)^{2}+\sigma\left(T_{n}\left(E_{n}, L\right)\right)^{2}} .
$$

In the following, the sub-index $10-G$ refers to the mean of the corresponding values for the $L_{3}$ and $L_{4}$ RPC layers, the 10-gaps RPC layers, e.g., $\sigma\left(T_{R P C_{10-G}}\right)$ is the mean of $\sigma\left(T_{R P C_{3}}\right)$ and $\sigma\left(T_{R P C_{4}}\right)$.

\subsection{Neutron selection}

Since one of the main goals of experiment S406 was the performance evaluation of the NeuLAND prototype, it was positioned just downstream from the magnet, to receive directly the neutrons created on the target (primary neutrons), while the RPC prototype was placed just downstream, next to NeuLand (see figure 4). Thus, in addition to being exposed to primary neutrons, the RPC was exposed to secondary particles: charged particles, photons and scattered neutrons, originated in interactions of the primary neutrons with the NeuLand prototype. In order to select primary neutrons hitting the RPC prototype, we used the NeuLand prototype as a veto. This requirement has a dramatic effect on the behaviour of the particles hitting the RPC, as illustrated in figure 5. There it is shown, in columns, from left to right, number of hits in each RPC layer (figures 5.a and 5.b), hit position distribution across the strips in each RPC layer (5.c and 5.d), hit position distribution 

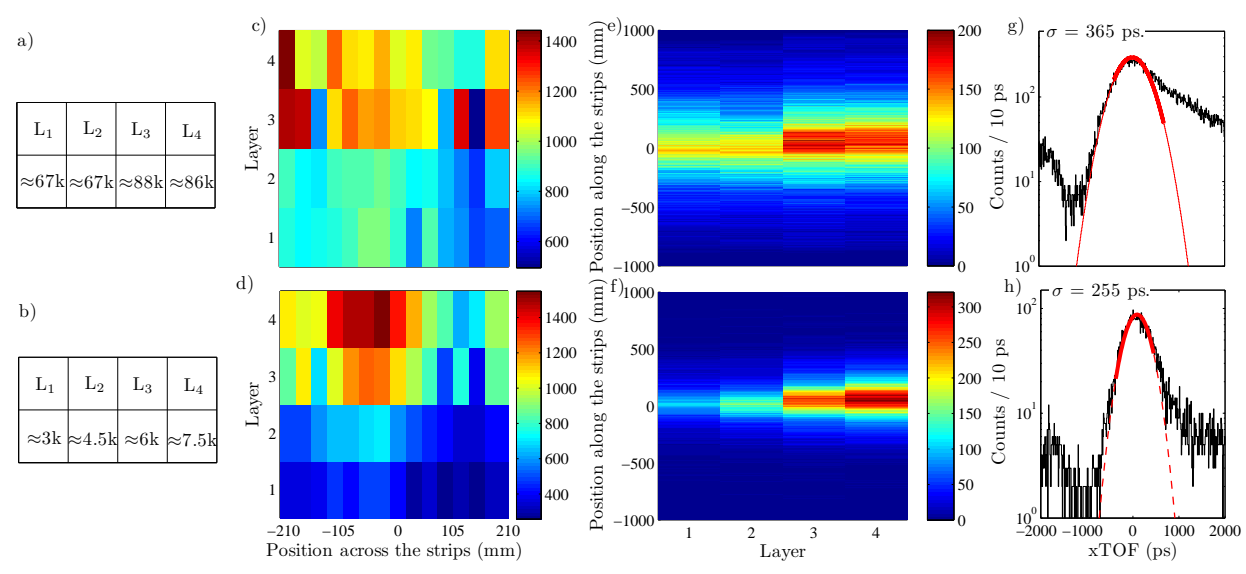

Figure 5. Behaviour of particles hitting the RPC. Upper plots show events coincident with signals on the NeuLand prototype while the lower plots show events without signals in NeuLand. In columns from left to right: a) and b), number of hits in each RPC layer; c) and d), hit position distribution across the strips in each RPC layer; e) and f), hit position distribution along the strips in each RPC layer; g) and h) $x T O F_{4}$ distribution for a $1500 \mathrm{AMeV}$ deuteron beam with the RPC at $6.03 \mathrm{~m}$ from the target.

along the strips in each RPC layer (5.e and 5.f) and the $\bar{n} T O F_{4}$ and $n T O F_{4}$ distributions (figures 5.g and 5.h) for a $1500 \mathrm{AMeV}$ deuteron beam with the RPC positioned at $6.03 \mathrm{~m}$ from the target. Upper plots show events in coincidence with signals in the NeuLand prototype, which should correspond to primary neutrons interacting in NeuLand with the consequent charged particles, photons and scattered neutrons reaching the RPC. In this case the hits in the RPC are dominated by charged particles, due to the higher detection efficiency for this kind of particles. ${ }^{3}$ Figure 5.a shows the number of hits in the RPC layers, which should be approximately similar since the charged particles cross all the RPC layers. The difference between layers 1, 2 (4-gaps) and 3, 4 (10-gaps) can be explained by their different efficiencies, see figure 3.c and [14]. Figure 5.c shows the hit position distribution across the strips, which should be approximately uniform since these particles leave NeuLand at different angles. Figure 5.e shows the hit position distribution along the strips and figure 5.g shows $\bar{n} T O F_{4}$ distribution with a huge (right) tail corresponding to slow particles. Lower plots show events without signals in the NeuLand prototype, which should correspond to primary neutrons going through this detector without interacting and then hitting the RPC. Figure 5.b shows the number of hits in the RPC layers, which should increase for deeper RPC layers since the neutrons create secondary charged particles which will be detected in the following layers. Figure 5.d shows the hit position distribution across the strips, which should peaked at the center of the RPC given that these neutrons fly in straight lines. Figure 5.f shows the hit position distribution along the strips, which is narrower when compared with figure 5.e. Finally, figure 5.h shows the $n \mathrm{TOF}_{4}$ distribution, which should be better compared with the charged particles case (figure 5.e) due to the smaller jitter in the flight path and velocity. Here, it is still appreciable a (right) tail in the distribution (slow particles), which means that the neutron selection is not perfect.

\footnotetext{
${ }^{3}$ It is expected, based on simulations [4], an efficiency of around $23 \%$ for $1500 \mathrm{MeV}$ neutrons, the efficiency for photons is negligible and efficiency for charged particles is close to 1 [14].
} 

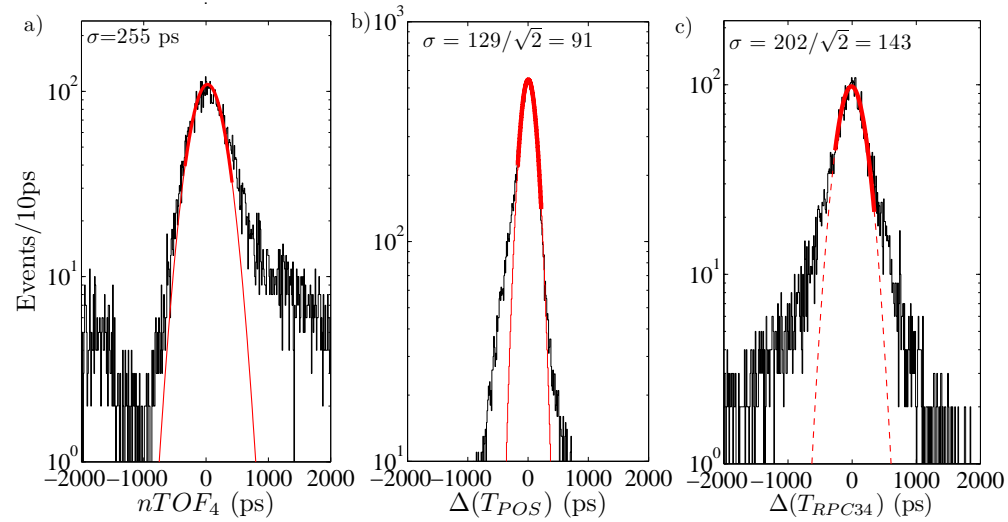

Figure 6. a) Time of flight distribution between the POSI detector and the $4^{\text {th }}$ RPC layer, $n T O F_{4}$, b) the $\Delta\left(T_{\mathrm{POS}}\right)$ distribution and c) $\Delta\left(T_{R P C_{34}}\right)$ for a $1500 \mathrm{AMeV}$ deuteron beam with the RPC at $6.03 \mathrm{~m}$ from the target.

\section{Results}

\subsection{RPC timing resolution}

Figure 6.a shows the neutron time of flight distribution between the POSI detector and the $4^{\text {th }}$ RPC layer, $n \mathrm{TOF}_{4}, 6 . \mathrm{b}$ the $\Delta\left(T_{\mathrm{POS}}\right)$ distribution and 6.c $\Delta\left(T_{R P C_{34}}\right)$ for a $1500 \mathrm{AMeV}$ deuteron beam with the RPC positioned at $6.03 \mathrm{~m}$. For this energy $\sigma\left(n T O F_{4}\right)$ is $255 \mathrm{ps}$, the contribution of the POSI detector, $\sigma\left(T_{\mathrm{POS}_{\mathrm{I}}}\right)$, is $91 \mathrm{ps}$ and the contribution of the $4^{\text {th }} \mathrm{RPC}$ layer, $\sigma\left(T_{R P C_{4}}\right)$, is $143 \mathrm{ps}{ }^{4}$ Table 1 shows these numbers also for $300,500,800 \mathrm{AMeV}$ at $6.03 \mathrm{~m}$ from the target and $1500 \mathrm{AMeV}$ at $12.97 \mathrm{~m}$ from the target and in addition $\sigma\left(n T O F_{i}\right)_{i=1,2,3}, \sigma\left(T_{n}\left(E_{n}, L\right)\right)$ and $\Delta\left(T_{R P C_{12}}\right)$. Table 1 only shows $\sigma\left(\Delta\left(T_{R P C_{i j}}\right)\right)$ for $i j=1,2$ and $i j=3,4$ (RPC layers with the same number of gaps). This is because the $\sigma\left(\Delta\left(T_{R P C_{i j}}\right)\right)$ calculated for layer of the same type (4-gaps or 10-gaps) is better when compared with $\sigma\left(\Delta\left(T_{R P C_{i j}}\right)\right)$ calculated for layer of different type (this behaviour is also visible in figure 3). We suspect that this is due to the presence of systematic errors with similar impact on layers of the same type, which are cancelled in the time difference between two layer of the same type $\left(\mathrm{L}_{1}-\mathrm{L}_{2}\right.$ or $\left.\mathrm{L}_{3}-\mathrm{L}_{4}\right)$ but they are not cancelled between layers of different type $\left(\mathrm{L}_{1}-\mathrm{L}_{3}, \mathrm{~L}_{2}-\mathrm{L}_{3}\right.$, ....), see [14] for a detailed explanation. $\sigma\left(\Delta\left(T_{R P C_{12}}\right)\right)$ is worse when compared with $\sigma\left(\Delta\left(T_{R P C_{34}}\right)\right)$ (this is also illustrated in figure 3 ). This is most probably due to the non-optimal HV working point and a possible construction defect on (one) of the 4-gaps RPC layers. $\sigma\left(n T O F_{i}\right)$ for $i=1,2$ is systematically worse compared with $\sigma\left(n T O F_{i}\right)$ for $i=3,4$. As the contribution of the RPC and POSI detectors is only significant at high energy and this effect is present at all energies, this could be related to the type of particles hitting the $1^{\text {st }}$ and $2^{\text {nd }}$ RPC layers, e.g., slow particles stopped in these layers. For this reason in the following only variables corresponding to the 10-gaps RPC layers are shown.

Figure 7.a shows the measured $\sigma\left(n T O F_{10-G}\right)$ (error bars correspond to $95 \%$ confidence interval) and $\sigma\left(n T O F_{10-G}\right)_{c a l}$ together with the three main contributions: $\sigma\left(T_{R P C_{10-G}}\right), \sigma\left(T_{\mathrm{POS}_{\mathrm{I}}}\right)$ and $\sigma\left(T_{n}\left(E_{n}, L\right)\right)$ as a function of energy (with the RPC at $6.03 \mathrm{~m}$ from the target) and 7.b as a function

\footnotetext{
${ }^{4} \sigma\left(T_{R P C_{4}}\right)$ is $70 \mathrm{ps}$ for $800 \mathrm{MeV}$ protons see figure 3 and reference [14].
} 
Table 1. Time of flight jitter between the POSI detector and the $i^{\text {th }}$ RPC layer, $\sigma\left(n T O F_{i}\right)$, and the different contributions for the four energies and two distances $L$ measured, $\sigma\left(T_{n}\left(E_{n}, L\right)\right), \sigma\left(T_{\mathrm{POS}_{\mathrm{I}}}\right)$ and $\sigma\left(T_{\mathrm{RPC}_{\mathrm{ij}}}\right)$.

\begin{tabular}{|c|c|c|c|c|c|c|c|c|c|}
\hline \multirow{2}{*}{ 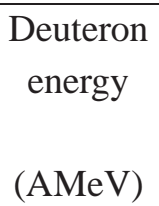 } & \multirow[t]{2}{*}{$\mathrm{L}$} & \multicolumn{4}{|c|}{$\sigma\left(n T O F_{i}\right)$} & \multirow{2}{*}{$\begin{array}{c}\sigma\left(T_{n}\left(E_{n}, L\right)\right) \\
(\mathrm{ps})\end{array}$} & \multirow{2}{*}{$\begin{array}{c}\sigma\left(T_{\mathrm{POS}_{\mathrm{I}}}\right) \\
(\mathrm{ps})\end{array}$} & \multicolumn{2}{|c|}{$\begin{array}{c}\sigma\left(\Delta\left(T_{R P C_{i j}}\right)\right) / \\
\sqrt{2}\end{array}$} \\
\hline & & $\begin{array}{l}i=1 \\
(\mathrm{ps})\end{array}$ & $\begin{array}{l}i=2 \\
\text { (ps) }\end{array}$ & $\begin{array}{l}i=3 \\
\text { (ps) }\end{array}$ & $\begin{array}{l}i=4 \\
\text { (ps) }\end{array}$ & & & $\begin{array}{c}i j=1,2 \\
(\mathrm{ps})\end{array}$ & $\begin{array}{c}i j=3,4 \\
(\mathrm{ps})\end{array}$ \\
\hline 1500 & 6.03 & 337 & 338 & 274 & 255 & 165 & 91 & 150 & 143 \\
\hline 800 & 6.03 & 482 & 473 & 438 & 405 & 391 & 171 & 178 & 138 \\
\hline 500 & 6.03 & 733 & 680 & 635 & 668 & 709 & 226 & 176 & 148 \\
\hline 300 & 6.03 & 1247 & 1331 & 1151 & 1194 & 1298 & 221 & 159 & 131 \\
\hline 1500 & 12.97 & 473 & 514 & 371 & 368 & 355 & 84 & 152 & 144 \\
\hline
\end{tabular}

of the distance $L$ for $1500 \mathrm{AMeV}$. The agreement between $\sigma\left(n T O F_{10-G}\right)$ and $\sigma\left(n T O F_{10-G}\right)_{c a l}$ in figure 7.a is quite good pointing to the contribution of the RPC is at a level of 140 ps independent of the energy of the neutron (average values of all $\sigma\left(\Delta\left(T_{R P C_{34}}\right)\right) / \sqrt{(2)}$ from table 1). The extrapolation of the $\sigma\left(n T O F_{10-G}\right)$ at $L=0, \sigma\left(T_{n}\left(E_{n}, 0\right)\right)=0$, taken from figure 7.b, dashed line, is $173 \mathrm{ps}$, that when subtracted quadratically the contribution of the POSI detector gives a value for the $\sigma\left(T_{R P C_{10-G}}\right)$ of $147 \mathrm{ps}$.

\section{Conclusions}

A prototype composed of four resistive plate chamber layers has been exposed to quasimonoenergetic neutrons produced from a deuteron beam of varying energy (300 to $1500 \mathrm{AMeV}$ ). Each RPC layer, with an active area of about $2000 \times 500 \mathrm{~mm}^{2}$ is made of modules built entirely from float glass electrodes. The particular aspect of this concept is that the glass electrodes that define the gas gaps within the RPC act also simultaneously as converter material to detect the re-

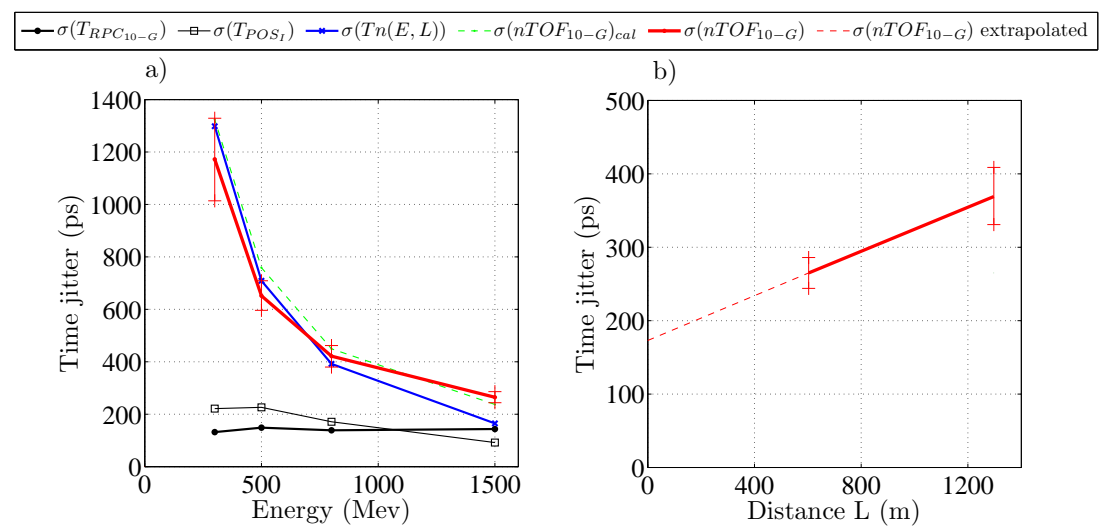

Figure 7. Time of flight jitter between the POSI detector and the average of $3^{\text {th }}$ and $4^{\text {th }}$ RPC layers $\sigma\left(T_{R P C_{10-G}}\right)$ a) as a function of energy, with the RPC at $6.03 \mathrm{~m}$ from the target, together with the three main contributions, and b) as a function of distance for a $1500 \mathrm{AMeV}$ deuteron beam. The error bars correspond to $95 \%$ confidence interval. 
lativistic neutrons. Measurements of the time of flight jitter of neutrons, in the mentioned energy range, point to a contribution of the RPC of the order of $150 \mathrm{ps}$ independent of the energy of the neutron. This value should be compared with the $\sigma_{t}<100 \mathrm{ps}$ requirement of the NEULAND proposal. But must be done carefully, since we strongly think that the result reported here should be interpreted as an upper limit since a better calibration of the RPC could be implemented (with more statistic available) and better data could be obtained exposing the RPC directly to a neutron beam (it is still visible a slow particle contamination in the time of flight distribution).

\section{Acknowledgments}

Work supported by the European Community FP7 Capacities, contract ENSAR no.262010, and Portuguese FCT project PTDC/FIS/114876/2009.

\section{References}

[1] R3B collaboration, http://www-win.gsi.de/r3b/Documents/R3B-TP-Dec05.pdf, December 2005.

[2] K. Boretzky et al., GSI Scientific Report, PHN-ENNA-EXP-60 (2012).

[3] LAND collaboration, T. Blaich et al., A Large area detector for high-energy neutrons, Nucl. Instrum. Meth. A 314 (1992) 136.

[4] J. Machado et al., Simulations of a new detection concept for high-energy neutrons based on timing RPCs, 2013 JINST 8 P07020.

[5] E. Cerron Zeballos et al., A new type of resistive plate chamber: The Multigap RPC, Nucl. Instrum. Meth. A 374 (1996) 132.

[6] D. Belver et al., Performance of the Low-Jitter High-Gain/Bandwidth Front-End Electronics of the HADES tRPC Wall, IEEE Trans. Nucl. Sci. 57 (2010) 2848.

[7] A. Gil et al., Distributed Low-Voltage System for the Front End of the HADES Timing RPC Wall, IEEE Trans. Nucl. Sci. 56 (2009) 382.

[8] A. Gil et al., The slow control system of the HADES RPC wall, Nucl. Instrum. Meth. A 661 (2012) S118.

[9] D. Belver et al., The HADES RPC inner TOF wall, Nucl. Instrum. Meth. A 602 (2009) 687.

[10] HADES collaboration, G. Agakishiev et al., The high-acceptance dielectron spectrometer HADES, Eur. Phys. J. A 41 (2009) 243 [arXiv: 0902.3478].

[11] J. Michel et al., The HADES DAQ System: Trigger and Readout Board Network, IEEE Trans. Nucl. Sci. 58 (2011) 1745.

[12] http://daq.gsi.de.

[13] J. Hoffmann, https://www.gsi.de/de/C/work/organisation/wissenschaftlich technologische abteilungen/ experimentelektronik/digitalelektronik/digitalelektronik/module/vme/vulom/vulom4.htm.

[14] J. Machado, J. Adamczewski-Musch, A. Blanco, K. Boretzky, P. Cabanelas et al., Performance of timing Resistive Plate Chambers with protons from 200 to 800 MeV, 2015 JINST 10 C01043.

[15] Z. Elekes et al., Simulation and prototyping of $2 \mathrm{~m}$ long resistive plate chambers for detection of fast neutrons and multi-neutron event identification, Nucl. Instrum. Meth. A 701 (2013) 86. 